\title{
A pillbox window with impedance matching sections for a W-band gyro-TWA
}

\author{
Liang Zhang, Craig R. Donaldson, Adrian W. Cross, and Wenlong He
}

\begin{abstract}
A mode-matching method was developed to design a pillbox-type millimeter wave window for a W-band gyrotron traveling wave amplifier. With additional impedance matching sections, the pillbox window was able to achieve $\mathbf{- 4 0} \mathrm{dB}$ reflection over a $10 \%$ frequency bandwidth. The manufactured pillbox window achieved a maximum reflection of $-15 \mathrm{~dB}$. The reasons for the difference between the simulated and measured reflections were investigated.
\end{abstract}

Index Terms - pillbox window, vacuum electron device, gyroTWA, microwave window.

\section{INTRODUCTION}

$\mathrm{M}$ icrowave windows are one of the key components in any microwave vacuum electron device (MVED). They separate the vacuum inside the device from the surrounding atmospheric pressure. Besides the requirements of ensuring and maintaning a complete hermetic seal with sufficient mechanical strength, the window also needs to have desirable microwave properties, such as low reflection and low insertion loss.

Microwave windows of different configurations have been developed for MVEDs used for different applications, such as the single-disc [1], multi-disc [2], pillbox [3-5] and Brewster types [6]. The pillbox window has a balanced performance between the bandwidth and the reflection. Its bandwidth is wider than the single-disc window and it has a less complicated geometry than the multi-disc and Brewster windows. The pillbox window has rectangular apertures at both input and output sides therefore, it is commonly used as an input microwave window for microwave amplifiers.

A W-band gyrotron traveling wave amplifier (gyro-TWA) was demonstrated at the University of Strathclyde. It was driven by an axis-encircling electron beam with a voltage of $55 \mathrm{kV}$ and current of $1.5 \mathrm{~A}$. With a helically corrugated interaction region the gyro-TWA was able to achieve a frequency bandwidth of about $10 \%$ [7]. The seed microwave signal was generated by a QuinStar solid-state source, and through a WR10 waveguide. It was fed into the interaction region by a pillbox window and a side-wall input coupler. The amplified microwave signal was $\mathrm{TE}_{11}$ mode in circular waveguide and with $\mathrm{kW}$ power level. It was converted into the Gaussian mode by a smooth-profiled horn [8] and then coupled out from the gyro-TWA through a three-disc microwave window [9].

In this paper, a demountable pillbox window for the W-band gyro-TWA as an input microwave window is presented. Its design goal is to achieve a minimum reflection over the frequency range of 90-100 GHz. The simulation method and

This work was supported by the Science and Technology Facilities Council (STFC) under Research Grant ST/P001890/1. L. Zhang (liang.zhang @ strath.ac. uk), C. R. Donaldson (craig.donaldson@strath.ac.uk), A. W. Cross (a.w.cross optimised design, manufacturing details and measurement results are also presented.

\section{SiMULATION AND OPTIMIZATION RESULTS}

A schematic drawing of the pillbox window is shown in Fig. 1(a). Several methods have been used to calculate its scattering parameters, including the equivalent circuit method [5, 10], the impedance matching approach [4] and the method of moments [11]. In our design, the mode-matching method that is able to take into account the effect of higher-order modes was used. As shown in Fig. 1(b), the window structure was divided into a series of basic components, including the straight waveguide, waveguide steps, interfaces between different media, and so on. Each component has its own characteristic scattering matrix (SM). The SM of the pillbox window can be cascaded by the $\mathrm{SM}$ of the individual components.

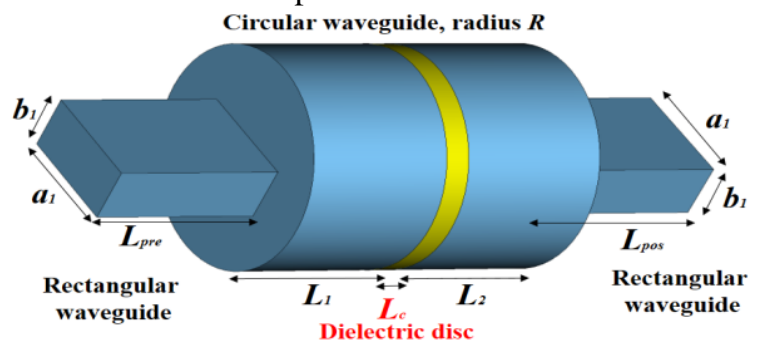

(a)

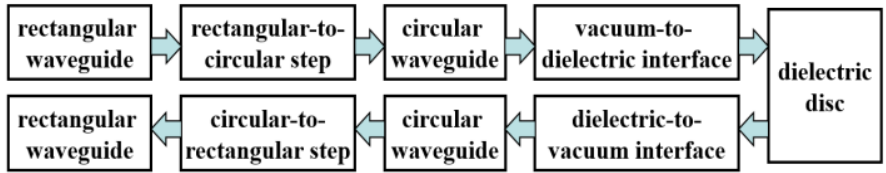

(b)

Fig. 1 (a) 3D schematic drawing of the pillbox type window (b) the connection of the components.

Among the basic components, the SMs of the straight waveguide and the dielectric disc are simplest and they can be expressed as $\mathrm{S}=\left[\begin{array}{cc}0 & S_{w} \\ S_{w} & 0\end{array}\right][12]$, where $S_{w}$ is a $N * N$ diagonal matrix with the elements $S_{n n}=\exp \left(-\beta_{n} L\right),(n=1,2, \ldots, N)$. $\beta_{n}$ is the propagation constant of the $n^{\text {th }}$ mode in the waveguide, and $L$ is the length of the waveguide. The most complicated component is the rectangular-to-circular waveguide step. Fortunately, the SM of a small rectangular-to-large circular waveguide step has been reported [13-15]. Duerr's thesis [13] gives the most detailed procedure based on the idea in [15],

@ strath.ac.uk), and W.He (w.he@ strath.ac.uk) are with Department of Physics, SUPA, University of Strathclyde, Glasgow, G4 0NG, Scotland, UK. 
where only the final result is given. The SM for the rectangularto-circular waveguide step is

$$
\begin{gathered}
\mathrm{S}=\left[\begin{array}{ll}
S_{11} & S_{12} \\
S_{21} & S_{22}
\end{array}\right] \\
S_{11}=\left(I+Z_{\alpha}^{1 / 2} M^{T} Z_{\beta}^{-1} M Z_{\alpha}^{1 / 2}\right)^{-1}\left(I-Z_{\alpha}^{1 / 2} M^{T} Z_{\beta}^{-1} M Z_{\alpha}^{1 / 2}\right) \\
S_{11}=2\left(\mathrm{I}+Z_{\alpha}^{1 / 2} M^{T} Z_{\beta}^{-1} M Z_{\alpha}^{1 / 2}\right)^{-1} Z_{\alpha}^{1 / 2} M^{T} Z_{\beta}^{-1 / 2} \\
S_{21}=Z_{\beta}^{-1 / 2} M Z_{\alpha}^{1 / 2}\left(I+S_{11}\right) \\
S_{22}=Z_{\beta}^{-1 / 2} M Z_{\alpha}^{1 / 2} S_{12}-I
\end{gathered}
$$

where $Z_{\alpha}, Z_{\beta}$ is the resistance matrix of the rectangular and circular waveguide, respectively. The $M$ matrix is determined by the geometry of the circular waveguide (radius $R$ ), the rectangular waveguide (lengths $a, b$ ) and their relative position (offset to the center $x_{1}, y_{1}$ ). The expression of $M$ can be found in section 2.2.4 in reference [13].

The SM of the discontinuity between different media can be extended from the paper [16] which deals with the TE modes in the rectangular waveguide. With a similar process, the SM for a circular waveguide with both TE and TM modes can be derived. The SM for TE mode is

$$
\mathrm{S}=\left[\begin{array}{cc}
\frac{f-g}{f+g} e^{-i \cdot 2 k_{z 1} z_{1}} & \frac{2 \sqrt{g f}}{g+f} e^{-i \cdot\left(k_{z 1}-k_{z 2}\right) z_{1}} \\
\frac{2 \sqrt{g f}}{g+f} e^{-i \cdot\left(k_{z 1}-k_{z 2}\right) z_{1}} & \frac{g-f}{f+g} e^{i \cdot 2 k_{z 2} z_{1}}
\end{array}\right]
$$

where $f=k_{z 1} / k_{z 2}, g=\mu_{2} / \varepsilon_{1} . k_{z 1}$ and $k_{z 2}$ are the wave number at both sides of the media interface, respectively.

The SM for TM mode is

$$
\mathrm{S}=\left[\begin{array}{cc}
\frac{f h-1}{f h+1} e^{-i \cdot 2 k_{z 1} z_{1}} & \frac{2 \sqrt{f h}}{f h+1} e^{-i \cdot\left(k_{z 1}-k_{z 2}\right) z_{1}} \\
\frac{2 \sqrt{f h}}{f h+1} e^{-i \cdot\left(k_{z 1}-k_{z 2}\right) z_{1}} & \frac{1-f h}{f h+1} e^{i \cdot 2 k_{z 2} z_{1}}
\end{array}\right]
$$

where $h=\varepsilon_{2} / \varepsilon_{1}$. In general, the interface position was chosen as $z_{1}=0$ to simplify the calculation.

The SM of the whole pillbox window can be obtained by cascading all the SMs of the individual component from left to right. The equation to cascade two SMs can be found in [12]. For the pillbox design, a few simplifications can be used to speed up the simulation by an order of magnitude. (1) The matrix $M$ in Eq. 6 was derived for general structures with an offset to the center $x_{1}, y_{1} \neq 0$. However, the offset would excite higher order modes which are undesired. $M$ can be greatly simplified when applying $x_{1}=y_{1}=0$. (2) From previous experience, it was found that a symmetric structure is able to achieve a minimum reflection due to less geometry discontinuities. A mirror boundary can be therefore set in the middle of the cavity (the position of half thickness of the dielectric disc). This not only reduces the free geometry parameter number but also reduce the calculation time. (3) There is no mode conversion at the media interface. Therefore the SMs of the straight waveguides including the dielectric disc, and the media interface all are diagonal. The matrix cascading operation can be downgraded to vector operation. (4) The rectangular waveguides at both sides only affect the phase of the scattering parameters. Since only the amplitude information is needed, they do not have to be included in the calculation.

The mode-matching method provides fast and accurate results in a structure like the pillbox window. It allows full exploration of the parameter space. To find the optimal geometry that achieves a minimum reflection over the desired frequency range, a multi-objective optimization algorithm was employed. There were two objectives used in the optimization. One was to minimize the overall reflection, and the other was to minimize the maximum reflection. The evaluation functions for both objectives could be written as

$$
\left\{\begin{array}{l}
f_{1}(X)=\frac{1}{N} \sum_{F}^{N}\left(\operatorname{abs}\left(S_{11}(X, F)\right)-0\right)^{2} \\
f_{2}(X)=\operatorname{MAX}\left(\operatorname{abs}\left(S_{11}(X, F)\right)\right)
\end{array}\right.
$$

where $X$ is the parameter set to be optimized. $F$ is the frequency index in the calculated frequency range. $N$ is the number of frequencies to be calculated. $S_{11}$ is the absolute value of $S_{11}$ of the window.

In the optimization, the dielectric material was chosen as $99.4 \%$ pure $\mathrm{Al}_{2} \mathrm{O}_{3}$ the relative dielectric constant of this is 9.4. The thickness of the ceramic disc was chosen to be larger than $1 \mathrm{~mm}$ to allow sufficient mechanical strength. The optimization converged after about 5000 iterations, and took about 3 hours using an 8-core i7-5960X CPU with 16 simulations running in parallel. The structure of the optimum pillbox window is shown in Fig. 2(a). It was able to achieve a maximum reflection of lower than $-30 \mathrm{~dB}$ over the frequency range of $90-100 \mathrm{GHz}$.

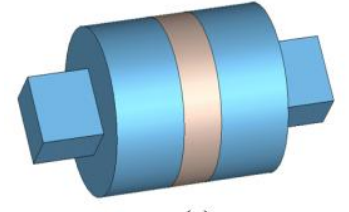

(a)

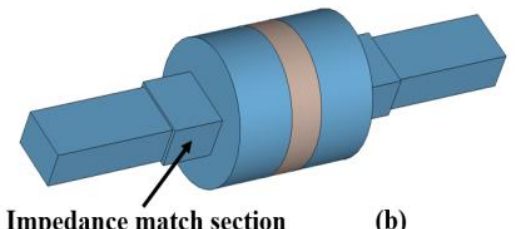

(b)
Fig. 2 The structures of the pillbox windows: (a) without and (b) with IMS.

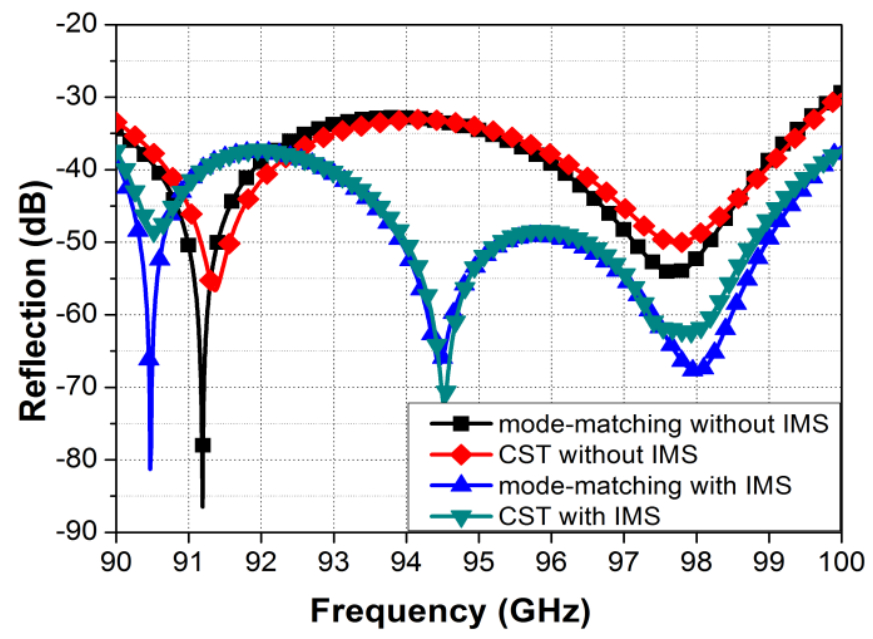

Fig. 3 The optimized reflections of the pillbox windows without and with IMS.

The overall reflection of the pillbox window could be further reduced by adding impedance matching sections (IMS) between the rectangular and circular waveguides. The theory of IMS can be found in [17]. More IMSs would normally result in lower reflection. However, that would also increase the machining difficulty. Therefore, two identical IMSs at two sides were added as shown in Fig. 2(b). The dimensions of the IMS could be estimated by using the equations in [17] at the center frequency. To achieve a minimum reflection over the 
desired frequency range, the multi-objective optimization was carried out. The IMS was regarded as a rectangular waveguide step, whose scattering matrix can be found in [18]. Therefore the reflection of the pillbox window with IMS can be simulated using the mode-matching method.

The optimization results shown that with the IMS, the overall reflection of the pillbox window could be reduced by another $5 \mathrm{~dB}$. The simulated microwave reflections from the structures by using mode-matching method and CST microwave studio are shown in Fig. 3.

\section{MANUFACTURING AND MEASUREMENT}

The designed pillbox window also contained waveguide tapers to the standard WR10 size. The whole structure was divided into a few parts. Each part was machined individually and then brazed together. To accurately assemble and locate the components in the brazing process, a few location holes with 1 $\mathrm{mm}$ diameter were machined on the parts. The window was designed to be demountable therefore it could be reused in other projects. One of its ends has a copper UG-387/U round flange to connect to the solid-state source in the experiment or a vector network analyzer (VNA) port for measurement. On the vacuum side, a stainless steel flange was used. It had the same size and threaded holes with UG-387/U flange for measurement. It also had a custom CF-like knife edge to seal the vacuum.

The thickness of the ceramic disc was $1.33 \mathrm{~mm}$. It was initially brazed to a thin copper sleeve. This helps to minimize or eliminate the possible fillet on the ceramic to copper joint caused by the brazing material which has significant detrimental effect on the reflection. The copper sleeve was then brazed with the other parts of the assembly at a lower temperature. The microwave window after brazing is shown in Fig. 4.

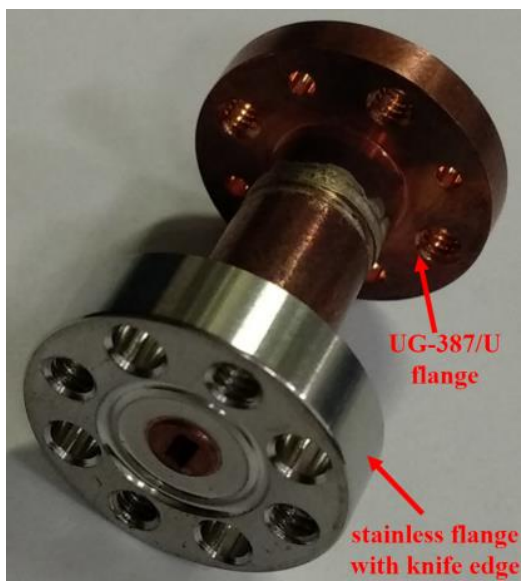

Fig. 4 The pillbox window after brazing assembly.

The pillbox window was leak tested before it was measured. The custom knife edge was able to maintain a vacuum down to 1E-9 mbar. The millimeter wave properties of the pillbox window was measured by a vector network analyzer and the results is shown in Fig. 5. The reflection of the pillbox window in $90-100 \mathrm{GHz}$ range was about $-15 \mathrm{~dB}$. The Ohmic loss of the window was about $-0.5 \mathrm{~dB}$, which was mainly due to the relatively thick ceramic disc.
The numerical optimization was also used to investigate the reason that caused the discrepancies between the design and measurement. In this case the measured reflection result was used as the optimization goal and random tolerances were applied to the geometry and dielectric constant of the ceramic disc. The optimization results indicated that it was able to match the measurement results by applying tolerances to the geometry, as shown in Fig. 5. The differences were mainly caused by the tolerances from parameters $b_{1}, L_{1}, L_{2}$ (marked in Fig. 1) as well as the lengths of the impedance matching sections. Other parameters, such as $R, a_{1}, L_{c}$ and dielectric constant have less contribution in this case. Further simulations shown that $L_{1}, L_{2}$ are the most sensitive parameters. With a change of $\pm 0.07 \mathrm{~mm}$, the reflection increased to $-17 \mathrm{~dB}$ in the frequency range of 90 - $100 \mathrm{GHz}$. The change of $b_{1}$ and the lengths of the IMS had similar degrees of effect on the refection level. A tolerance level of $\pm 0.1 \mathrm{~mm}$ in their dimensions still kept the maximum reflection under $-25 \mathrm{~dB}$ over $90-100 \mathrm{GHz}$. Further improvement could be achieved by reducing the machining tolerance of $L_{1}$ and $L_{2}$.

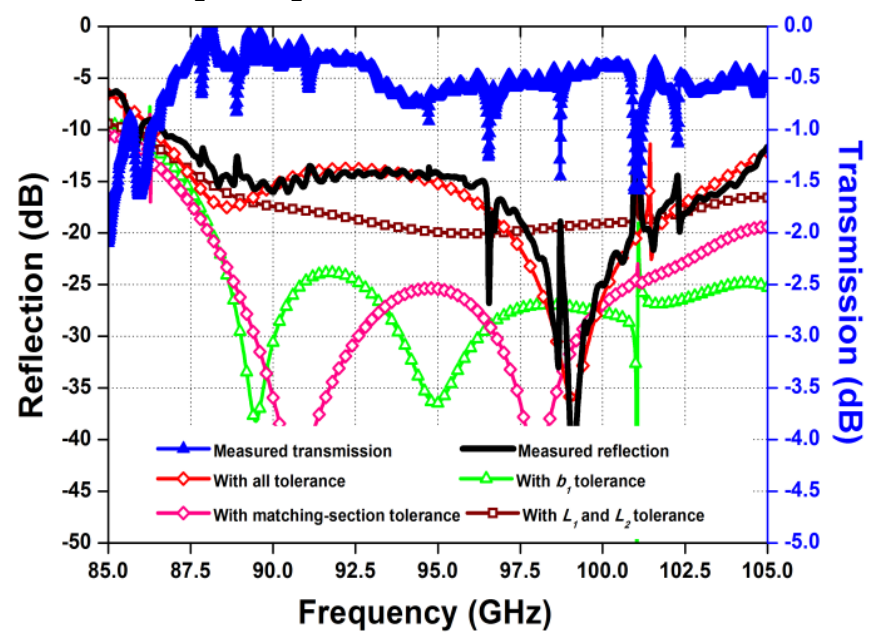

Fig. 5 measurement result of the pillbox window.

\section{CONCLUSION}

This paper reported the design, manufactory and measurement of a pillbox-type microwave window for a $\mathrm{W}$ band gyrotron traveling wave amplifier as an input window. By using the mode-matching method, the fast simulation allowed full exploration on a large number of geometries. With additional impedance matching sections, better bandwidth and reflection characteristics was achieved. The designed pillbox window was manufactured as a demountable component so that it could be reused in other devices. In the measurement, the reflection was about $-15 \mathrm{~dB}$ and the transmission loss was about $-0.5 \mathrm{~dB}$. The difference between the measurement and simulations was mainly caused by the errors in the length of the circular waveguide.

\section{REFERENCES}

M.-C. Lin, H.-M. Chung, and A. Burke, "An analytical approach for the fast design of high-power waveguide windows," Wave Motion, vol. 37, no. 2, pp. 183-188, Feb. 2003. DOI: 10.1016/S01652125(02)00073-2 
[2] C. R. Donaldson, W. He, L. Zhang, and A. W. Cross, "A W-Band Multi-Layer Microwave Window for Pulsed Operation of GyroDevices," IEEE Microw. Wireless Compon. Lett., vol. 23, no. 5, pp. 237-239, May 2013. DOI: 10.1109/LMWC.2013.2251619

[3] A. M. Cook, C. D. Joye, T. Kimura, E. L. Wright, and J. P. Calame, "Broadband 220-GHz Vacuum Window for a Traveling-Wave Tube Amplifier," IEEE Trans. Electron Devices, vol. 60, no. 3, pp. 12571259, March 2013. DOI: 10.1109/TED.2012.2232929

[4] H. Ao, H. Asano, F. Naito, N. Ouchi, J. Tamura, and K. Takata, "Impedance matching of pillbox-type RF windows and direct measurement of the ceramic relative dielectric constant," Nucl. Instrum. Methods. Phys. Res. A, vol. 737, pp. 65-70, Feb. 2014. DOI: 10.1016/j.nima.2013.11.048

[5] H. Nakatsuka, N. Yoshida, and I. Fukai, "Three-dimensional analysis of a vacuum window connected to waveguide," IEEE Trans. Plasma Sci., vol. 16, no. 4, pp. 416-422, Apr. 1988. DOI: $10.1109 / 27.3853$

[6] Y. Zhang, L. Zhang, W. He, C. R. Donaldson, P. McElhinney, A. D. R. Phelps, and A. W. Cross, "Design and Measurement of a W-Band Brewster Window," IEEE Microw. Wireless Compon. Lett., vol. 25, no. 12 , pp. 826-828, Dec. 2015 . DOI: 10.1109/LMWC.2015.2495110

[7] W. He, C. R. Donaldson, L. Zhang, K. Ronald, A. D. R. Phelps, and A. W. Cross, "Broadband Amplification of Low-Terahertz Signals Using Axis-Encircling Electrons in a Helically Corrugated Interaction Region," Phys. Rev. Lett., vol. 119, no. 18, p. 184801, Oct. 2017. DOI: 10.1103/PhysRevLett.119.184801

[8] L. Zhang, W. He, C. R. Donaldson, G. M. Smith, D. A. Robertson, R. I. Hunter, and A. W. Cross, "Optimization and Measurement of a Smoothly Profiled Horn for a W-Band Gyro-TWA," IEEE Trans. Electron Devices, vol. 64, no. 6, pp. 2665-2669, June 2017. DOI: 10.1109/TED.2017.2687949

[9] C. R. Donaldson, P. McElhinney, L. Zhang, and W. He, "WideBand HE11 Mode Terahertz Wave Windows for Gyro-Amplifiers," IEEE Transactions on Terahertz Science and Technology, vol. 6, no. 1, pp. 108-112, June 2016. DOI: 10.1109/TTHZ.2015.2495221

[10] S. Liu, "A RF window for broadband millimeter wave tubes," Int. J. Infrared Millimeter Waves, vol. 17, no. 1, pp. 121-126, Jan. 1996. DOI: $10.1007 / \mathrm{BF} 02088187$

[11] H. Arai, N. Goto, Y. Ikeda, and T. Imai, "An Analysis of a Vacuum Window for Lower Hybrid Heating," IEEE Trans. Plasma Sci., vol. 14, no. 6, pp. 947-954, June 1986. DOI: 10.1109/TPS.1986.4316645

[12] C. Tak Sum and T. Itoh, "Generalized Scattering Matrix Method for Analysis of Cascaded and Offset Microstrip Step Discontinuities," IEEE Trans. Microwave Theory Techn., vol. 34, no. 2, pp. 280-284, Feb. 1986. DOI: 10.1109/TMTT.1986.1133323

[13] E. K. Duerr, "Millimeter-wave Integrated Horn Antennas," Master thesis, Department of Electrical Engineering and Computer Science, Massachusetts Institute of Technology, 1998.

[14] R. Keller and F. Arndt, "Rigorous modal analysis of the asymmetric rectangular iris in circular waveguides," IEEE Microw. Guid. Wave Lett., vol. 3, no. 6, pp. 185-187, June 1993. DOI: 10.1109/75.219809

[15] R. H. MacPhie and W. Ke-Li, "Scattering at the junction of a rectangular waveguide and a larger circular waveguide," IEEE Trans. Microwave Theory Techn., vol. 43, no. 9, pp. 2041-2045, Sep. 1995. DOI: $10.1109 / 22.414538$

[16] M.-C. Lin, "A Multilayer Waveguide Window for Wide-Bandwidth Millimeter Wave Tubes," Int. J. Infrared Millimeter Waves, vol. 28, no. 5, pp. 355-362, May 2007. DOI: 10.1007/s10762-007-9207-y

[17] R. C. Johnson, "Design of Linear Double Tapers in Rectangular Waveguides," IRE Transactions on Microwave Theory and Techniques, vol. 7, no. 3, pp. 374-378, July 1959. DOI: 10.1109/TMTT.1959.1124594

[18] L. Christie and P. Mondal, "Mode Matching Method for the Analysis of Cascaded Discontinuities in a Rectangular Waveguide," Procedia Computer Science, vol. 93, pp. 251-258, Jan. 2016. DOI: 10.1016/j.procs.2016.07.208 